\title{
Lymph Node Metastasis of Squamous Cell Carcinoma from an Unknown Primary in the Upper and Middle Neck: Impact of ${ }^{18} \mathrm{~F}$-Fluorodeoxyglucose Positron Emission Tomography/Computed Tomography
}

\author{
Philippe B. Deron, ${ }^{1}$ Katrien M. Bonte, Hubert F. Vermeersch, and Christophe Van de Wiele ${ }^{2}$
}

\begin{abstract}
Purpose: The purpose of this study was to assess the potential of ${ }^{18} \mathrm{~F}$-fluorodeoxyglucose positron emission tomography/computed tomography (FDG-PET/CT) imaging for detection of the primary tumor and its impact on treatment planning in patients presenting with cancer of unknown primary and squamous cell carcinoma (SCC)-positive cervical lymph nodes of the upper and middle neck.

Methods: The study population consisted of 18 consecutive patients with biopsy-proven SCC involving lymph nodes of the upper and middle neck region and negative conventional diagnostic procedures with regard to the location of the primary. All patients underwent FDG-PET/CT according to a standard procedure in search for the primary, unidentified tumor.

Results: In none of the patients FDG-PET/CT was able to indicate a primary tumor localization. Although FDGPET/CT did identify all sites of known lymph node involvement, neither additional sites of lymph node involvement nor sites of distant metastases were identified. Accordingly, FDG-PET/CT did not impact patient treatment planning.

Conclusions: In this series, including patients suffering from lymph node metastases by an SCC of unknown primary in the upper and middle neck, FDG-PET/CT was unable to identify a primary tumor. In addition, FDGPET/CT did not modify the treatment planning in any of the patients studied.
\end{abstract}

Key words: computed tomography, head and neck, positron emission tomography, squamous cell carcinoma, unknown primary

\section{Introduction}

U $p$ to $15 \%$ of cancer patients present with metastasis from a cancer of unknown primary (CUP). ${ }^{1}$ Median survival of patients with CUP and an identified primary site subsequently treated with specific therapy is on average 23 months versus 12 months for those patients in whom the primary site is not identified. ${ }^{2}$ Thus, detection of the primary tumor in CUP patients is of paramount importance.

Cervical lymph nodes are a common site of involvement in CUP patients. The most commonly encountered histological diagnosis of CUP with cervical lymph node involve- ment is squamous cell carcinoma (SCC), followed by adenocarcinoma. ${ }^{3-5}$ Cervical lymph node metastases of SCC origin in the upper and middle neck are generally attributed to cancers of the head and neck. ${ }^{6}$ For these patients, routine workup generally consists of a physical examination, biopsy or excision of the enlarged lymph nodes, computed tomography (CT) and/or magnetic resonance imaging, and panendoscopy. ${ }^{7}$ With this routine workup, the primary SCC remains undetected in $\sim 2 \%$ of patients.

Previous reports on the usefulness of ${ }^{18} \mathrm{~F}$-fluorodeoxyglucose positron emission tomography (FDG-PET) in CUP patients with negative clinical and conventional diagnostic

\footnotetext{
${ }^{1}$ Department of Head and Neck Surgery, University Hospital Ghent, Ghent, Belgium.

${ }^{2}$ Department of Nuclear Medicine, University Hospital Ghent, Ghent, Belgium.

Address correspondence to: Philippe B. Deron; Department of Head and Neck Surgery, University Hospital Ghent; De Pintelaan, 185, B-9000 Gent, Belgium

E-mail: philippe.deron@ugent.be
} 
procedures suggest that FDG-PET may detect up to 5\%-43\% of the primary tumors missed by clinical and morphological evaluation. ${ }^{7,9-20}$ These figures, however, were reported in series that included patients with CUP and extracervical metastases as well as varying cancer types, for example, SCC, adenocarcinoma, melanoma, large cell carcinoma, plasmocytoma, etc., and the diagnostic performance of FDGPET was not determined separately for cervical versus extracervical CUP presentations and as a function of the histological subtype. Further, in several of these studies, the PET methodology was either not standardized or inadequate and most of these studies were conducted using FDG-PET and not FDG-PET/CT, which might have resulted in a higher rate of false-positive findings and sensitivity.

In this study, the potential of FDG-PET/CT imaging for detection of the primary tumor and its impact on treatment planning in patients presenting with CUP and SCC-positive cervical lymph nodes of the upper and middle neck were assessed.

\section{Patients and Methods}

Between November 2002 and November 2007, patients presenting to the Department of Head and Neck Surgery of University Hospital of Gent, Belgium, with metastatic SCC of the upper and middle neck were included in this study.

All patients had routine workup with medical history and physical examination including rigid endoscopy of the nasal cavity and nasopharynx and flexible fiber-endoscopy of the oropharynx, hypopharynx, and larynx. Contrast-enhanced high-resolution computed tomography of the neck (slice thickness of $3 \mathrm{~mm}$ ) and the thorax (spiral scanning) was performed in all patients. Fine-needle aspiration cytology or histology of the metastatic lymph nodes proved the presence of metastatic SCC. In all selected patients, neither clinical examination nor computer tomography was able to put forward a possible primary tumor localization.

Next, all patients underwent a full-body FDG-PET/CT to guide eventual biopsies in the following planned panendoscopy under general anesthesia. FDG-PET/CT was performed according to the standard protocol at the Ghent University Hospital. The patients fasted at least 4 hours prior to intravenous injection of F-18 FDG $(3.7 \mathrm{MBq} / \mathrm{kg}$ body weight). Blood glucose levels were checked prior to the injection of F-18 FDG and did not exceed $140 \mathrm{mg} / \mathrm{dL}$. If not contraindicated, iodinated intravenous contrast (iohexol, $350 \mathrm{mg}$ iodine/mL [GE Healthcare, Diegem, Belgium], or iomeprol, $400 \mathrm{mg}$ iodine $/ \mathrm{mL}$ [Bracco Imaging Europe, Waver, Belgium]) was administered immediately before CT scanning. Patients were imaged with the Gemini PET-CT imaging system (Philips Co., Cleveland, $\mathrm{OH}$ ), which consists of a gadolinium oxyorthosilicate full-ring PET scanner with $5.0 \mathrm{~mm}$ spatial resolution and a 16-slice helical CT scanner. After a 60-minute uptake period, during which patients were instructed to rest silently, images were acquired. First, a CT surview $(30 \mathrm{~mA}, 120 \mathrm{kV}$, FOV $500 \mathrm{~mm}$, collimation $0.75 \mathrm{~mm}$ ) was performed from the base of the skull through the mid thigh. This was followed by a low-dose CT $(30 \mathrm{~mA}, 120 \mathrm{kV}$, FOV $500 \mathrm{~mm}, 0.5$ seconds rotation time, pitch 0.9 , collimation $16 \times 1.5 \mathrm{~mm})$ and a high-quality CT $(150 \mathrm{~mA}, 120 \mathrm{kV}$, FOV $500 \mathrm{~mm}, 0.5$ seconds rotation time, pitch 0.9 , collimation $16 \times 1.5 \mathrm{~mm}$, slice thickness and increment $5 \mathrm{~mm}$ ) without specific breath-holding instructions and arms at the side of the torso. PET scanning was performed immediately after acquisition of the CT images, without changing the patient position. Images were acquired from the base of the skull to the proximal half of the femora. PET images were reconstructed using an iterative 3D-RAMLA (Row Action Maximum Likelihood Algorithm) provided by the vendor. Low-dose CT data were used for attenuation correction.

Two skilled nuclear medicine physicians and radiologists who were unaware of the clinical findings evaluated FDGPET/CT results. In cases of discrepancy, the FDG-PET/CT interpretation was reached by consensus. In case of positive FDG-PET/CT findings, surgery or biopsy was required to confirm that positive findings were indeed related to the presence of a primary tumor. If the FDG-PET/CT findings were negative, long-term follow-up was used to confirm the initial negative findings.

\section{Results}

Eighteen consecutive patients were included in the study. There were 16 men and 2 women with a mean age of 58.8 years (range: $45.8-78.7$ years). The metastatic lymph nodes were located in neck regions II or III or overlapped both regions. Nine lymph nodes were smaller than $3 \mathrm{~cm}$ in diameter, six were larger than $3 \mathrm{~cm}$ but smaller than $6 \mathrm{~cm}$ in diameter, and three were larger than $6 \mathrm{~cm}$ in diameter. Nine patients presented with solitary metastatic lymph node involvement, whereas in the remaining 9 patients multiple lymph nodes were involved. According to the UICC 1997 TNM classification, the N-stage of the study patients was N1 in 3, N2a in 4, N2b in 7, N2c in 1, and N3 in 3 patients.

Although all known sites of lymph node involvement were visualized, in none of the patients included did FDGPET/CT examinations demonstrate a primary tumor. Additionally, in none of the patients additional lymph nodes or distant metastases that could have impacted the therapeutic decision-making were detected.

Patients were treated with a combination of therapies according to the oncologic guideline of the Ghent University Hospital: 4 patients had primary radiotherapy, 9 patients had surgery and adjuvant postoperative radiotherapy, 2 patients had surgery and postoperative concomitant radiochemotherapy (platinum based), and 3 patients had concomitant radiochemotherapy (platinum based). The radiation therapy was intensity modulated with a total dose of 70 Gy and covered the complete mucosa of the upper airway. The chemotherapy was based on cisplatinum at a dose of $100 \mathrm{mg} / \mathrm{m}^{2}$ and given on days 1,22 , and 43 of the radiotherapy.

With a median follow-up time of 32 months, 10 patients remained alive without evidence of disease, 6 patients died of their disease (all due to distant metastatic disease), and 2 patients were lost to follow-up. Follow-up did not reveal a primary tumor in any of the patients studied, neither by regular clinical examination nor by CT scanning.

\section{Discussion}

Finding the primary tumor site in patients presenting with SCC involving cervical lymph nodes of the upper and middle neck presents a diagnostic and therapeutic dilemma. To minimize cosmesis while curing the tumor in these patients, 
surgery, with or without selective radiation therapy, is the treatment of choice. ${ }^{1-3}$ However, if the primary tumor is not identified, usually, generalized radiation therapy is administered, which includes the naso-, oro-, and hypopharynx, sometimes with debilitating effects. Further, survival is significantly better for those patients in whom the primary can be identified.

Following thorough clinical examination and morphological imaging, the primary tumor remains undetected in $<2 \%$ of patients with cervical lymph node metastases of an SCC, explaining the limited number of patients included in this series and other studies available on this topic. ${ }^{8} \mathrm{Al}-$ though, in this series, FDG-PET/CT identified all known neck metastases, confirming the FDG avidity of SCC, this imaging modality proved to be unable to identify a primary tumor in any of the patients under study. This finding may be attributed to several causes. First, the size of the lesion as well as the degree of FDG uptake may have been too low and thus, because of the limited spatial resolution and related partial volume effect of PET systems, the lesion may have passed undetected. ${ }^{21}$ More recently, time-of flight PET machines were introduced in the market. ${ }^{22,23}$ These machines allow for the registration of the actual time difference between the arrival of the annihilation photons in the detectors. Incorporation of this information in the reconstruction algorithm improves the spatial resolution. Thus, in the future, studies comparing conventional PET with TOF-PET for the detection of SCC CUP in the upper and middle neck may prove of interest. Second, it has been hypothesized that angiogenic incompetent primary tumors may remain very small, under the detection limit of the system, and even undergo apoptosis following the metastatic process. ${ }^{24}$ In this regard, it was found that in cases of SCC involving the head and neck, the primary tumors, uninvolved mucosa, and metastatic lymph nodes had significantly lower total blood vessels counts than the nonmetastatic cervical lymph nodes. ${ }^{25}$ Further, in the same study, neoangiogenesis proved much lower in metastatic lymph nodes when compared with the primary tumor, suggesting that the growth of tumors in the metastatic lymph nodes is independent of angiogenesis; lymph node metastases occupy well-vascularized lymph nodes, and lymph sinuses are rich in nutrients. Obviously, if the lesions are no longer there because of spontaneous apoptosis, it cannot be detected.

At first glance, the findings of the present study are in sharp contrast with results reported in a recent meta-analysis performed by Kwee et al. on the potential of combined PET/ CT for the detection of CUP. ${ }^{26}$ In this meta-analysis, overall FDG-PET/CT was able to detect $37 \%$ of primary tumors in patients with CUP, respectively, with a sensitivity and specificity of $84 \%$. The most commonly detected location of the primary tumor by FDG-PET/CT was the lung $(33 \%)$, and the most common locations of false-positive findings were the lung and oropharynx. Importantly, the sensitivity proved heterogeneous across studies, and subgroup analysis was unable to offer additional information in this regard. In 7 of 11 studies included, the location of the sites of metastases was cervical as well as extracervical, and in 3 of 4 of the remaining studies, minimally three different histological subtypes of metastatic sites were included. Only one study included, by Fakhry et al., reported on a series of 22 patients suffering from SCC involving lymph nodes from CUP. ${ }^{27}$ In this series, adopting a similar strategy to ours, FDG-PET/CT detected 4 primary tumors that were not detected by routine staging. Because of limited patient details in this study, direct comparison of the present study with the study by Fakhry et al. is not possible. For instance, it would have been interesting to identify whether sites of lymph node involvement, respectively, upper, middle versus lower cervical, were different between both studies and also whether the extent of lymph node involvement was different between those patients in whom a primary was detected versus those in whom no primary was identified.

Aside from the search for an unidentified primary, in the present study, the potential therapeutic impact of FDG-PET/ CT was also addressed. Four studies have previously reported on the therapeutic impact of FDG-PET/CT in CUP patients. ${ }^{27-30}$ In these studies, FDG-PET/CT modified therapy in $18.2 \%-60 \%$ of patients. For instance, in the series by Fakhry et al. in 4 patients in whom FDG-PET/CT identified the primary tumor, the size of the radiation fields could be reduced and thus also morbidity. ${ }^{27}$ These reports are in sharp contrast with the findings of the present study; aside from the lack of detection of a primary tumor, no additional sites of lymph node involvement or distant metastases that could have influenced the treatment planning were detected.

Finally, whether or not FDG-PET/CT also impacts patient outcome in CUP patients is an issue that needs to be explored. To date, only one study, by Fencl et al., suggested that FDG-PET/CT in CUP patients might indeed affect outcome. $^{31}$ In this series, the survival rate of CUP patients with at least one hypermetabolic lesion proved significantly lower when compared with the remaining CUP patients. Additional studies addressing this issue are warranted.

\section{Conclusions}

To conclude, in this series, including only patients suffering from lymph node metastases by an SCC of unknown primary in the upper and middle neck, FDG-PET/CT proved to be unable to identify the primary tumor. In addition, FDGPET/CT did not modify the treatment planning in any of the patients studied.

\section{Disclosure Statement}

There are no conflicts of interest to be declared by any of the authors.

\section{References}

1. Schapira DV, Jarrett AR. The need to consider survival, outcome and expense when evaluating and treating patients with unknown primary carcinoma. Arch Intern Med 1995; 155:2050.

2. Raber MN, Faintuch J, Abbruzzese JL, et al. Continuous infusion 5-fluorouracil, etoposide and cis-diamminedichloroplatinum in patients with metastatic carcinoma of unknown primary origin. Ann Oncol 1991;2:519.

3. Engzell U, Jacobsson PA, Sigurdson A, et al. Aspiration biopsy of metastatic carcinoma in lymph nodes of the neck. Acta Otolaryngol 1971;72:138.

4. Nordstrom DG, Tewfik HH, Latourette HB. Cervical lymph node metastases from an unknown primary. Int J Radiat Oncol Biol Phys 1979;5:73. 
5. Leipzig B, Winter ML, Hokanson JA. Cervical node metastases of unknown origin. Laryngoscope 1981;91:593.

6. Regelink G, Brouwer J, de Bree R, et al. Detection of unknown primary tumours and distant metastases in patients with cervical metastases: Value of FDG-PET versus conventional modalities. Eur J Nucl Med 2002;29:1024.

7. Issing WJ, Taleban B, Tauber S. Diagnosis and management of carcinoma of unknown primary in the head and neck. Eur Arch Otorhinolaryngol 2003;260:436.

8. Muir C. Cancer of unknown primary site. Cancer 1995;75 (Suppl):353.

9. Abbruzzese JL, Abbruzzese MC, Hess KR, et al. Unknown primary carcinoma: Natural history and prognostic factors in 657 consecutive patients. J Clin Oncol 1994;6:1272.

10. Pavlidis N. Forty years experience of treating cancer of unknown primary. Acta Oncologica 2007;46:592.

11. Million RR, Cassisi NJ, Mancuso AA. The unknown primary. In: Million RR, Cassisi NJ (eds.), Management of Head and Neck Cancer: A Multidisciplinary Approach, 2nd edition. Philadelphia: Lippincott, 1994;311.

12. Grau C, Johansen LV, Jacobsen J, et al. Cervical lymph node metastases from unknown primary tumours: Results from a national survey by the Danish Society for Head and Neck Oncology. Radiother Oncol 2000;55:121.

13. Johansen J, Eigtved A, Buchwald C, et al. Implication of 18Ffluoro-2-deoxy-D-glucose positron emission tomography on the management of carcinoma of unknown primary in the head and neck: a Danish cohort study. Laryngoscope 2002;112:1578.

14. Rades D, Kühnel G, Wildfang I, et al. Localised disease in cancer of unknown primary (CUP): The value of positron emission tomography (PET) for individual therapeutic management. Ann Oncol 2001;12:1605.

15. Jungehülsing $M$, Scheidhauer $K$, Damm $M$, et al. $2\left({ }^{18} F\right)$ fluoro-2-deoxy-D-glucose positron emission tomography is a sensitive tool for the detection of occult primary cancer (carcinoma of unknown primary syndrome) with head and neck lymph node manifestation. Otolaryngol Head Neck Surg 2000;123:294.

16. Bohuslavizki KH, Klutmann S, Kröger S, et al. FDG PET detection of unknown primary tumours. J Nucl Med 2000; 41:816.

17. Miller FR, Hussey D, Beeram M, et al. Positron emission tomography in the management of unknown primary head and neck carcinoma. Arch Otolaryngol Head Neck Surg 2005;131:626.

18. Greven KM, Keyes JW, Williams DW, et al. Occult primary tumours of the head and neck: Lack of benefit from positron emission tomography imaging with 2-(F-18)fluoro-2-deoxyD-glucose. Cancer 1999;86:114.

19. Nieder C, Gregoire V, Ang K. Cervical lymph node metastases from occult squamous cell carcinoma: Cut down a tree to get an apple? Int J Radiat Oncol Biol Phys 2001;50:727.

20. Stöckli SJ, Mosna-Firlejczyk K, Goerres GW. Lymph node metastasis of squamous cell carcinoma from an unknown primary: Impact of positron emission tomography. Eur J Nucl Med Mol Imaging 2003;30:411.

21. Kwee TC, Basu S, Cheng G, et al. FDG PET/CT in carcinoma of unknown primary. Eur J Nucl Med Mol Imaging 2010; 37:635.

22. Mawlawi O, Townsend DW. Multimodality imaging: An update on PET/CT technology. Eur J Nucl Med Mol Imaging 2009;36 (Suppl.1):S15.

23. Surti S, Kuhn A, Werner ME, et al. Performance of Philips Gemini TF PET/CT scanner with special considerations for its time-of-flight imaging capabilities. I Nucl Med 2007; 48:471.

24. Naresh KN. Do metastatic tumours from an unknown primary reflect angiogenic incompetence of the tumour at the primary site?- a hypothesis. Med Hypotheses 2002;59:357.

25. Naresh KN, Nerurkar AY, Borges AM. Angiogenesis is redundant for tumour growth in lymph node metastases. Histopathology 2001;38:466.

26. Kwee TC, Kwee RM. Combined FDG-PET/CT for the detection of unknown primary tumours: Systematic review and meta-analysis. Eur Radiol 2009;19:731.

27. Fakhry N, Barberet M, Lussato D, et al. Role of [18F]-FDG PET-CT in the management of the head and neck cancers. Bull Cancer 2006;93:1017.

28. Bruna C, Journo A, Netter F, et al. On the interest of PET with 18F-FDG in the management of cancer of unknown primary (CUP). Med Nucl 2007;31:242.

29. Wartski M, Le Stanc E, Gontier E, et al. In search of an unknown primary tumor presenting with cervical metastases: Performance of hybrid FDG-PET-CT. Nucl Med Commun 2007;28:365.

30. Pelosi E, Pennone M, Deandreis D, et al. Role of whole body positron emission tomography computed/computed tomography scan with $18 \mathrm{~F}$-fluorodeoxyglucose in patients with biopsy proven tumor metastases from unknown primary site. Q J Nucl Med Mol Imaging 2006;50:15.

31. Fencl P, Belohlavek O, Skopalova M, et al. Prognostic and diagnostic accuracy of [18F]FDG-PET/CT in 190 patients with carcinoma of unknown primary. Eur J Nucl Med Mol Imaging 2007;34:1783. 
This article has been cited by:

1. Kuruva Manohar, Bhagwant Rai Mittal, Anish Bhattacharya, Amod Gupta. 2012. Orbital Metastases as Presenting Sign of Lung Carcinoma: Detection of Primary Malignancy and Disease Burden by F-18 FDG PET/CT. Nuclear Medicine and Molecular Imaging . [CrossRef] 